\title{
Open Multi-Access Edge Computing and Distributed Mobility Management with RINA
}

\author{
Eduard Grasa*, Miguel Ponce de Leon ${ }^{\ddagger}$, Sven van der Meer ${ }^{\dagger}$, Diego Lopez ${ }^{\S}$ and Miguel Tarzan* \\ ${ }^{*}$ RINA research area, Fundacio i2CAT, \{eduard.grasa, miquel.tarzan\}@i2cat.net \\ ${ }^{\dagger}$ Network Management Lab, Ericsson, Athlone, Co. Westmeath, Ireland., sven.van.der.meer@ericsson.com \\ ${ }^{\ddagger}$ Kodacall, Arclabs, Carriganore, Waterford, Ireland., miguel@kodacall.com \\ $\S$ Telefonica Investigation y Desarrollo S.A., diego.r.lopez@telefonica.com
}

\begin{abstract}
MEC or Multi-Access Edge Computing is a network service delivery architecture that enables cloud computing capabilities at the edge of the access network. Realisation of the MEC vision is challenging with current network technologies: service discovery and routing, secure network slicing and distributed mobility management require complex capabilities in the network. This demo features an OMEC use case realised via RINA, the Recursive Network Architecture. RINA provides interesting properties for OMEC, due to its support for multi-homing, mobility and slicing without special protocols, and security as a first class element of the architecture.
\end{abstract}

\section{INTRODUCTION AND MOTIVATION}

Many network operators foresee a future where fronthaul load will be considerably higher, with the increasing rise of mobile data usage and location-based applications (social, analytics, video, AR/VR, etc.). There will be a requirement for local storage, local compute and networking processing of edge services in the mobile network, enabling cloud capabilities at closer proximities to the mobile user. This view point is forcing operators to seek new architectural directions, by disaggregating and virtualising the radio access network (RAN) and the evolved packet core (EPC), and then seeking the best way to have digital services processed at the edge of the network domain.

MEC or Multi-Access Edge Computing is a network service delivery architecture that enables cloud computing capabilities at the edge of the access network. Initially conceived in the context of cellular access networks, MEC is migrating towards a multi-access approach, in which the defining property of a MEC service is its availability through the network edge and not the specific access technology. MEC enables access to services with a lower latency, facilitating the delivery of location-based services. It also enables operators to reduce the traffic processed by the core network.

However the realisation of the MEC vision is challenging with current network technologies. Traditional mobility management approaches in cellular network are designed to forward user traffic to specific anchor points at the core network, since they assume services accessed by the user will be colocated within the anchor point or reachable through it. Service discovery is also an important part of MEC, since users may be accessing MEC and non-MEC services at the same time. The network must route each request to the appropriate service endpoint - locating it within the network - and

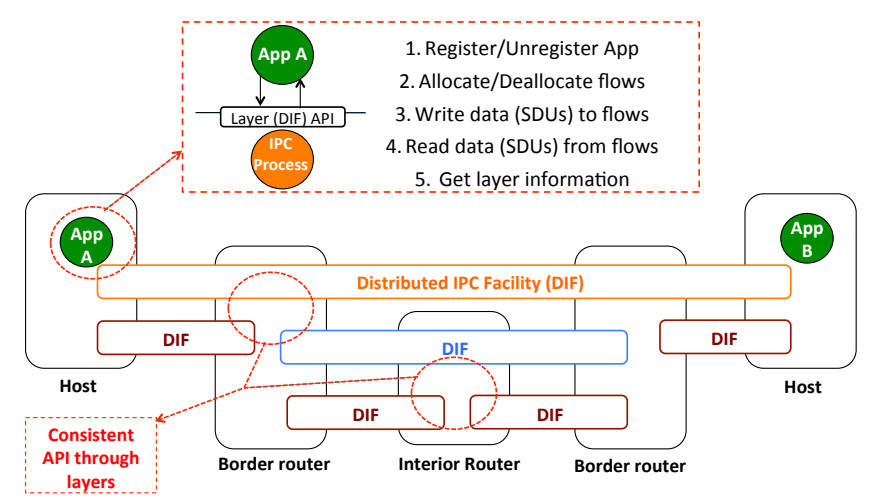

Fig. 1. Layers in RINA (down), and grouping of functions of protocol machine in the layer(IPC Process, up)

provision the required mechanisms to ensure that the service is delivered with enough quality. QoS and service continuity during handover are also important points for the performance of MEC service delivery. A number of organisations are already working to solve some of these issues, ETSI MEC [1] or IEEE OMEC being representative examples.

The value proposition showcased in this demo is the application of RINA to an OMEC scenario. RINA [2], the Recursive InterNetwork Architecture, is a fundamental network architecture that relies on the premise that networking is nothing more and nothing less than distributed Inter Process Communication (IPC). RINA decomposes networks into layers of generic protocols that can be configured via policies to optimally serve their operational requirements. As seen in Figure 1, in RINA there is a single type of layer - called a DIF, Distributed IPC Facility - that repeats as many times as needed by the network designer. RINA provides the right properties for materialising the OMEC vision: it supports multi-homing without the need of special protocols, it can carry out distributed mobility management, it supports application naming and discovery through multiple networks [3], it supports network slicing built-into the architecture (a slice can be seen as a QoS cube within a DIF or sometimes a dedicated DIF with specific QoS requirements) [4] and each DIF can be configured with the adequate security policies for authentication, access control and confidentiality [5]. 


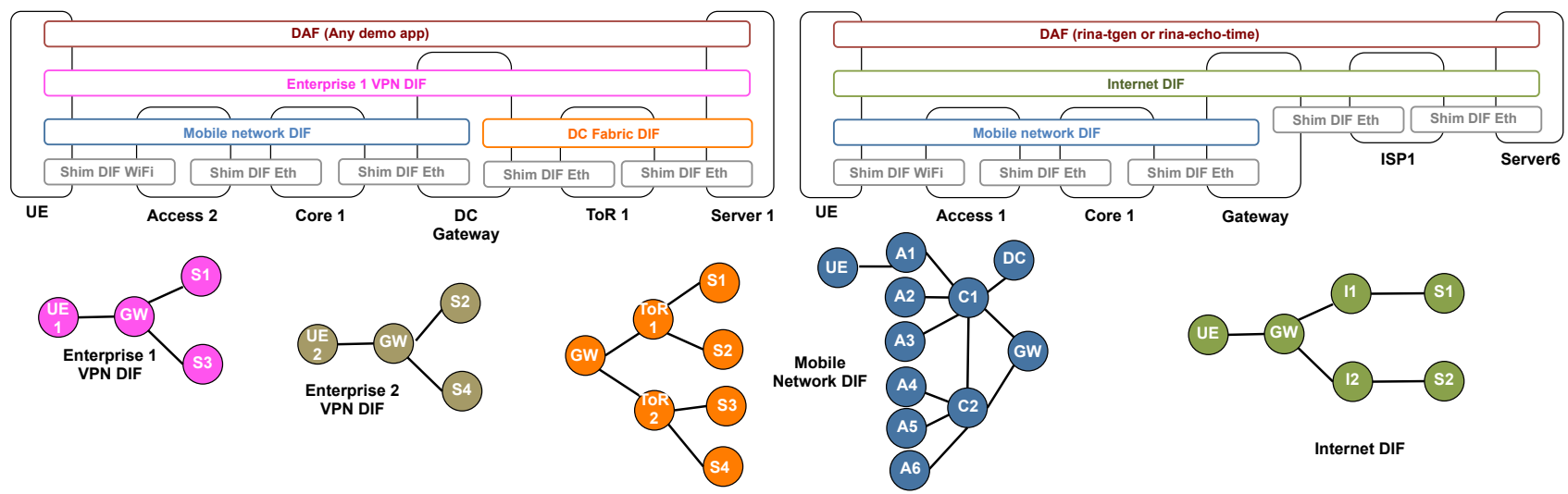

Fig. 2. Layer diagrams: UE accessing a service on the Internet (right) and a service in the service provider cloud (left)

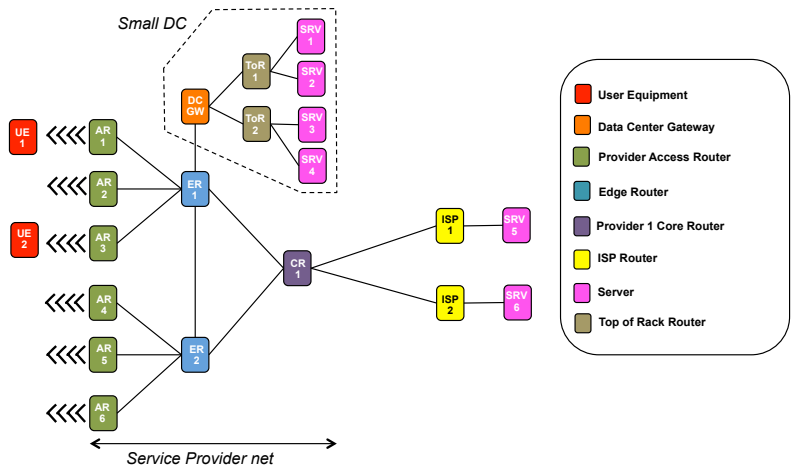

Fig. 3. Physical systems present in the demo

\section{DEMO SCENARIO AND STORYBOARD}

Figure 3 shows a view of the physical systems involved in the demo. Six Raspberry Pis will play the role of WiFi Access Routers (ARs) of the provider network. The ARs are divided into two groups of three, each one connected to an Edge Router (ER). Both ERs are connected to a Core Router (CR), which connects the service provider network to the Internet via two ISP routers. Each ISP router is directly connected to a Server. One ER is connected to a Data Centre (DC) gateway, which provides access to a small service-provider owner DC with four servers. All these systems (except for the Raspberry Pis) will be deployed as KVM/QEMU Virtual Machines (VMs). A couple of User Equipment (UE) hosts will access services deployed on servers reachable via the Internet and/or the service provider edge data-centre. All systems have RINA capabilities via the IRATI open source RINA implementation [6].

Layer diagrams are shown in Figure 2. The right part of the Figure shows the layers (DIFs) involved in the case when the UE accesses an application via the Internet DIF. The Mobile Network DIF (in blue) features distributed mobility management, allowing the UE to move through the access network without impacting service continuity, while still providing shortest path routing. A recorded, smaller-scale demonstration of distributed mobility management in RINA is available online at [7].
When the application requests a flow to a service, the RINA system will locate that service (through which DIF is it available), make the UE join the Internet DIF and allocate the flow to the destination application. If the service is available locally at the service provider DC, RINA will also locate the service and in this case make the UE join an Enterprise VPN DIF (which involves authentication and access control), and finally allocate the flow to the application hosted at the DC.

The demo will feature the two UEs moving through the access network and periodically allocating flows to different application instances hosted at either the service provider DC or at one of the two servers connected to the ISP routers. Each UE belongs to a different company. Access to each enterprise DIF will require the UEs to present the adequate credentials for going through authorisation and access control. Although the demo assumes a complete RINA scenario to better showcase the potential of the technology, deployments where RINA is hidden from applications and transports IP traffic are also possible. In a MEC scenario, RINA could just be deployed at the operator network and data centres, leaving User Equipment and application servers at DCs untouched.

\section{ACKNOWLEDGMENT}

This work is partly funded by the European Commission through the H2020 ARCFIRE project (Grant 687871).

\section{REFERENCES}

[1] E. M. ISG, "Mobile edge computing (mec); framework and reference architecture," ETSI MEC GS 003, March 2016.

[2] J. Day, I. Matta, and K. Mattar, "'Networking is IPC": A Guiding Principle to a Better Internet," in Proceedings of the 2008 ACM CoNEXT Conference, 2008.

[3] E. Trouva, E. Grasa, J. Day, and S. Bunch, "Layer discovery in rina networks," in Computer Aided Modeling and Design of Communication Links and Networks (CAMAD), 2012 IEEE 17th International Workshop on, 2012, pp. 368-372.

[4] P. T. Neil Davies, "Challenges of network slicing," IEEE SDN Newsletter, January 2017.

[5] E. Grasa, O. Rysavy, O. Lichtner, H. Asgari, J. Day, and L. Chitkushev, "From protecting protocols to protecting layers: designing, implementing and experimenting with security policies in rina," IEEE ICC 2016, Communications and Informations Systems Security Symposium, 2016.

[6] "Irati rina implementation for linux o/s," https://github.com/IRATI/stack.

[7] "Irati distributed mobility management demo at ieee omec workshop," http://ict-arcfire.eu/index.php/research/videos/. 